\title{
A MICROPOWER LEARNING VECTOR QUANTIZER FOR PARALLEL ANALOG-TO-DIGITAL DATA COMPRESSION
}

\author{
Jeremy Lubkin and Gert Cauwenberghs \\ Department of Electrical and Computer Engineering \\ The Johns Hopkins University, Baltimore, MD 21218-2686 \\ E-mail: gert@bach.ece.jhu .edu
}

\begin{abstract}
An analog VLSI architecture for learning vector quantization (LVQ), with on-chip adaptation and dynamic storage of the analog templates, is presented. The architecture extends to Fuzzy ART and Kohonen self-organizing maps through digital programming. The analog memory and adaptive element of the LVQ cell comprise $6 \mathrm{MOS}$ transistors and one capacitor, and provide for robust selfrefresh of the dynamic analog storage. Total cell size including distance and adaptive computations is $80 \times 70$ lambda in scalable MOSIS technology. Experimental results from a fabricated $16 \times 16$ cell prototype in $2 \mu \mathrm{m} \mathrm{CMOS}$ are included.
\end{abstract}

\section{INTRODUCTION}

Vector quantization (VQ) [1] is widely used for digital encoding of vectorial analog signals, with applications to pattern recognition and data compression in vision, speech and beyond. Learning VQ (LVQ) augments coding with adaptive on-line construction of the template codebook, for optimal performance under changing input statistics. Neural variants on LVQ are Adaptive Resonance Theory (ART) [2] and Kohonen self-organizing maps, among others.

In its basic form, the implementation of VQ involves a search among a set of vector templates for the one which best matches the input vector, according to a given distance metric. Learning continually adjusts the best matching templates towards the input.

Several analog VLSI vector quantizers and their variants have been developed in recent years, e.g. [3]-[9]. The $16 \times 16$ VQ chip presented here is implemented in current-mode BiCMOS technology for low-power operation, and integrates learning as well as long-term dynamic capacitive storage of the analog templates using an incremental partial refresh scheme [12].

\section{ARCHITECTURE}

The architecture of the learning vector quantizer is shown in Figure 1 . The core contains an array of $16 \times 16$ distance estimation cells interconnecting rows of templates with columns of input components. Each cell constructs the distance between one component $x_{j}$ of the input vector $\mathbf{X}$ and the corresponding component $y^{i}{ }_{j}$ of one of the template vectors $\mathbf{y}^{i}$,

$$
d\left(x_{j}, y_{j}^{i}\right)=\left|x_{j}-y^{i}{ }_{j}\right|^{\nu}, i, j=1 \ldots 16,
$$

This work was supported by ARPA/ONR MURI N00014-95-1-0409, and NSF Career Award MIP-9702346. Chip fabrication was provided through MOSIS.

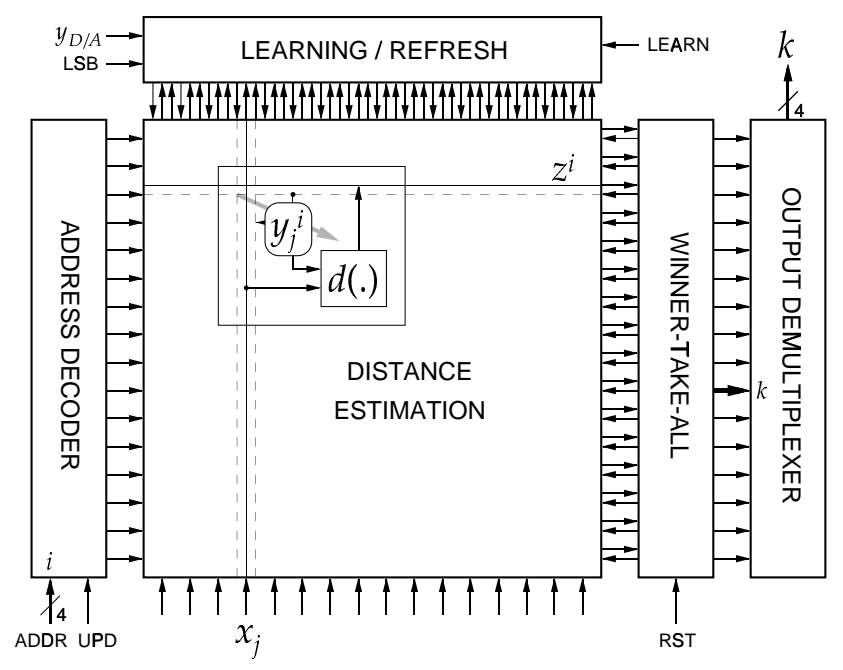

Figure 1: Parallel VLSI architecture for analog-to-digital vector quantization $(V Q)$, including template learning and refresh functions.

using a mean absolute difference (MAD) distance metric, with norm $\nu=1$. The component-wise distance is accumulated across inputs along template rows

$$
z^{i}=\sum_{j=1}^{16} d\left(x_{j}, y_{j}^{i}\right), \quad i=1 \ldots 16
$$

and presented to a winner-take-all (WTA), which selects the single winner

$$
k^{\mathrm{WTA}}=\arg \min _{i} z_{i} .
$$

Learning VQ is performed by selecting the winning template $k$ and producing an incremental update $\Delta \mathbf{y}^{k}$ in the stored vector $\mathbf{y}^{k}$ towards the input vector,

$$
\Delta y_{j}^{k}=\lambda\left(x_{j}-y_{j}^{k}\right) .
$$

where $\lambda$ determines the rate of adaptation. In the case of Kohonen self-organizing maps [3], the neighbors of the winner, $k=k^{\mathrm{WTA}} \pm 1$, are also adjusted according to (4) to preserve topological ordering in the digital coding.

The implemented architecture is also capable of performing fuzzy adaptive resonance [2] classification and learning, for stable 


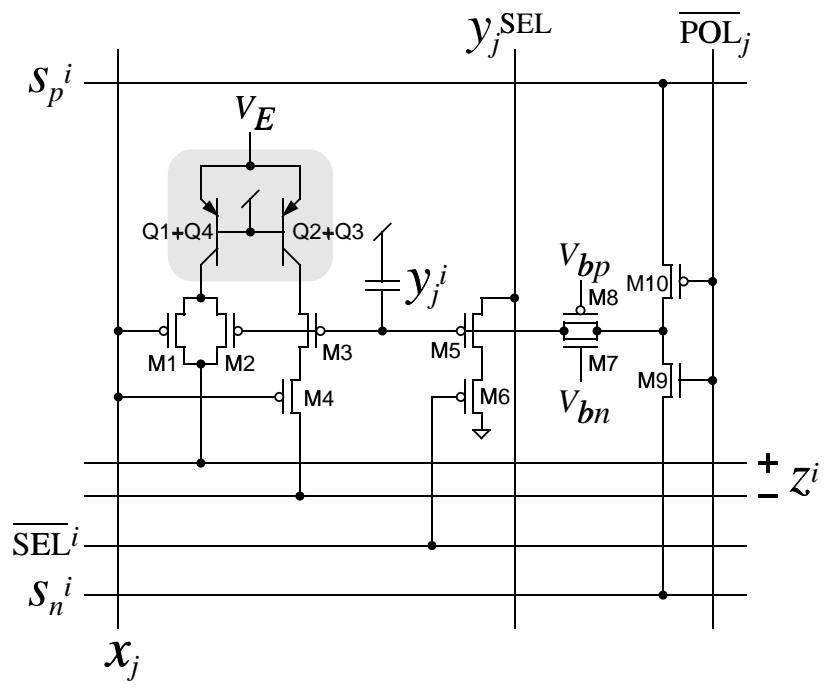

Figure 2: Circuit schematic of the VQ distance estimation cell, with integrated learning and template refresh. The dashed inset indicates a matched double pair of lateral bipolar transistors.

coding with control over the granularity of the template classes, by reconfiguring the chip. This amounts to modifying (1) into a fuzzy minimum, and corresponding changes to the learning updates (4).

\section{VLSI IMPLEMENTATION}

The circuits are implemented in current-mode BiCMOS technology, with MOS transistors operated in subthreshold for low-power dissipation. Lateral bipolar transistors offer full compatibility with standard CMOS processes.

\subsection{Distance Estimation}

The circuit diagram of the distance estimation cell is shown in Figure 2. The principle to construct the mean absolute error (1) measure between $x_{j}$ and $y^{i}{ }_{j}$ is similar to the approach in [7], except here operated continuous-time and in current-mode. The measure (1) is decomposed as the difference between the maximum and the minimum of $x_{j}$ and $y^{i}{ }_{j}$, accumulated separately onto two wires $z^{i}+$ and $z^{i}-$ and differentially combined outside of the array. The differential distance computation is performed by modulating the Early effect (collector conductance) of a matched (double) pair of bipolar transistors Q1-Q4 and Q2-Q3, by means of MOS transistors M1, M2, M3 and M4 connected as source followers. Parallel and series connections in the source followers yield the maximum and minimum of $x_{j}$ and $y_{j}^{i}$, respectively, in the output currents.

A centroid geometry, shown in Figure 3, is used for improved matching between the bipolar transistor currents that supply the differential output. By combining collector outputs in pairs $C_{1}+C_{4}$ and $C_{2}+C_{3}$, systematic variations and gradients in geometry are cancelled to first order. Matching is important, since the collector conductance is relatively small. The Early effect is maximized by using a minimum length geometry for the base, equaling the minimum length of an MOS transistor.

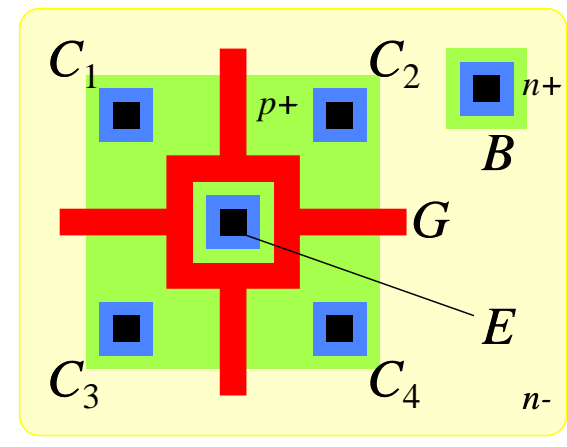

Figure 3: Centroid geometry of the matched double pair of lateral bipolar transistors, in conventional n-well CMOS technology.

The winner-take-all (WTA) is implemented as a variation on the standard current-mode design in $[10,11]$ with the addition of a triggered voltage-mode output stage for improved settling accuracy and speed [7].

\subsection{Learning and Refresh}

Transistors M5 through M10 implement the incremental update $\Delta y^{i}{ }_{j}$, when the cell is selected either for refresh, or for learning (when $k^{\text {WTA }} \equiv i$ ). The update $\Delta y^{i}{ }_{j}$ is constant amplitude, variable polarity. A constant-amplitude discrete update is easier to implement than a continuous update, and gives superior results in the presence of analog imprecisions in the implementation [13]. The polarity $\overline{\mathrm{POL}}_{j}$ of the update $\Delta y^{i}{ }_{j}$ is precisely implemented by means of a binary controlled charge pump [12]. The charge pump is free of switch charge injection parasitics, by avoiding clock signals on the MOS gates that couple capacitively into the storage capacitor. $V_{b n}$ and $V_{b n}$ are biased deep in subthreshold for precisely controlled increments and decrements as small as $10 \mu \mathrm{V}$. The timing of the update (and selection of the template) is performed by means of signals $S_{n}^{i}$ and $S_{p}^{i}[13]$.

The update polarity is computed externally to the array, by circuitry common for all cells on the same column, shown at the top of Figure 1. This arrangement is most space efficient since only one row of cells needs to be updated at once. A global LEARN signal selects the mode of operation, learning or refresh.

Figure 4 shows the simplified schematic of the learning cell, one per column of VQ distance cells. The circuit receives the selected analog template value $y^{i}{ }_{j}$, which is used to generate the update polarity $\overline{\mathrm{POL}}_{j}$ and supply it to the selected distance cell. When a distance cell is selected, switch M6 is closed and transistors M5-M6 along with M11-M13 implement a comparator. The update is performed in the cell according to $\overline{\mathrm{POL}}_{j}$ by activating the signals $S_{n}^{i}$ and $S_{p}^{i}$ for the entire row of selected cells.

In learning mode (LEARN三1), the polarity $\overline{\mathrm{POL}}_{j}$ is computed by comparing $y^{k}{ }_{j}$ with the input $x_{j}$, yielding a modified update (4)

$$
\Delta y_{j}^{k}=\mu \operatorname{sgn}\left(x_{j}-y^{k}{ }_{j}\right) \text {. }
$$

where $\mu$ is the constant-amplitude adaptation rate. In refresh mode (LEARN $\equiv 0), y^{k}{ }_{j}$ is compared with an external reference signal $y_{\mathrm{D} / \mathrm{A}}$ to construct a binary quantization function used for partial incremental refresh [12]. As in [14], the binary quantization $Q$ of $y^{k}{ }_{j}$ is obtained by retaining the least significant bit (LSB) of analogto-digital (A/D) conversion of $y^{k}{ }_{j}$. A single-slope sequential A/D 


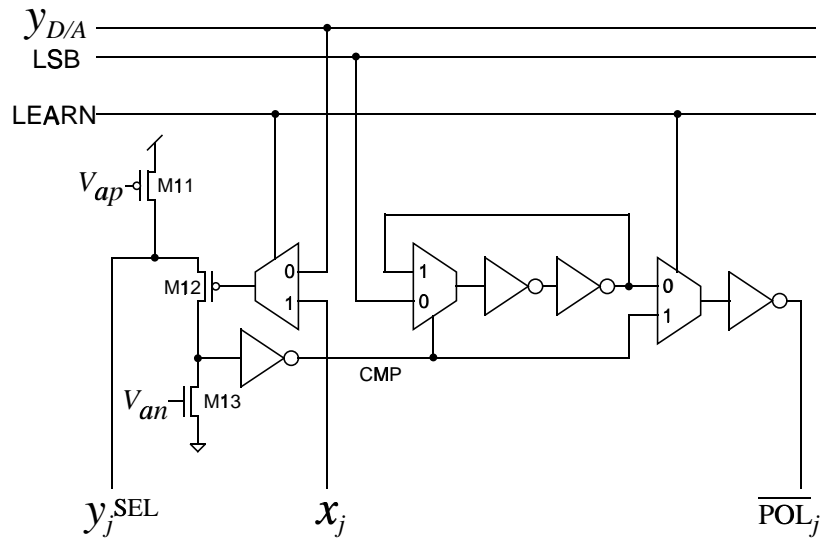

Figure 4: Simplified schematic of the learning and refresh circuitry, in common for a column of VQ cells. Analog multiplexers are implemented with complementary CMOS switches.

is implemented for simplicity, using a D/A signal on $y_{\mathrm{D} / \mathrm{A}}$, ramped up in discrete steps synchronously with the alternating LSB. When the comparator flips sign, the instantaneous LSB value is sampled and latched to generate the update polarity $Q\left(y^{k}{ }_{j}\right)$, producing an update [12]

$$
\Delta y_{j}^{k}=-\mu Q\left(y_{j}^{k}\right)
$$

\section{EXPERIMENTAL RESULTS}

A micrograph of the $16 \times 16$ learning vector quantizer, implemented in $2 \mu \mathrm{m}$ CMOS technology, is shown in Figure 5. The chip is fully functional, and dissipates $2 \mathrm{~mW}$ from a $5 \mathrm{~V}$ supply at a $10 \mathrm{ksample} / \mathrm{s}$ parallel data rate.

The experimental distance metric is illustrated in Figure 6, obtained by sweeping one of the 16 inputs while fixing the other inputs to the template values. The inverting offset of the metric is intended to interface the current output with the winner-take-all, which searches for maximum current $-z^{i}$. A fuzzy ART metric [2] is obtained by bypassing one of the two differential currents from the array.

Results for dynamic refresh of the templates at 32-level quantization are shown in Figure 7, obtained by observing the drift in stored voltage level on one of the cells over 1000 refresh cycles, for 300 different initial values of the voltage $y^{i}{ }_{j}$. The corresponding drift without refresh would have been tens of volts. Refresh tests across the entire array demonstrated long-term storage with minor level drifts over more than 1000 cycles. Further improvements in resolution and stability can be achieved, at some expense in silicon area, by using the A/D/A quantizer in [14].

Learning tests which validate the functionality of the learning vector quantizer with adaptive updates according to (5) are illustrated in Figure 8. The asymmetry in charging rate for upward and downward transitions is caused by transistor mismatch. Further experiments are currently underway to characterize system-level performance, for applications in speech coding and reconstruction.

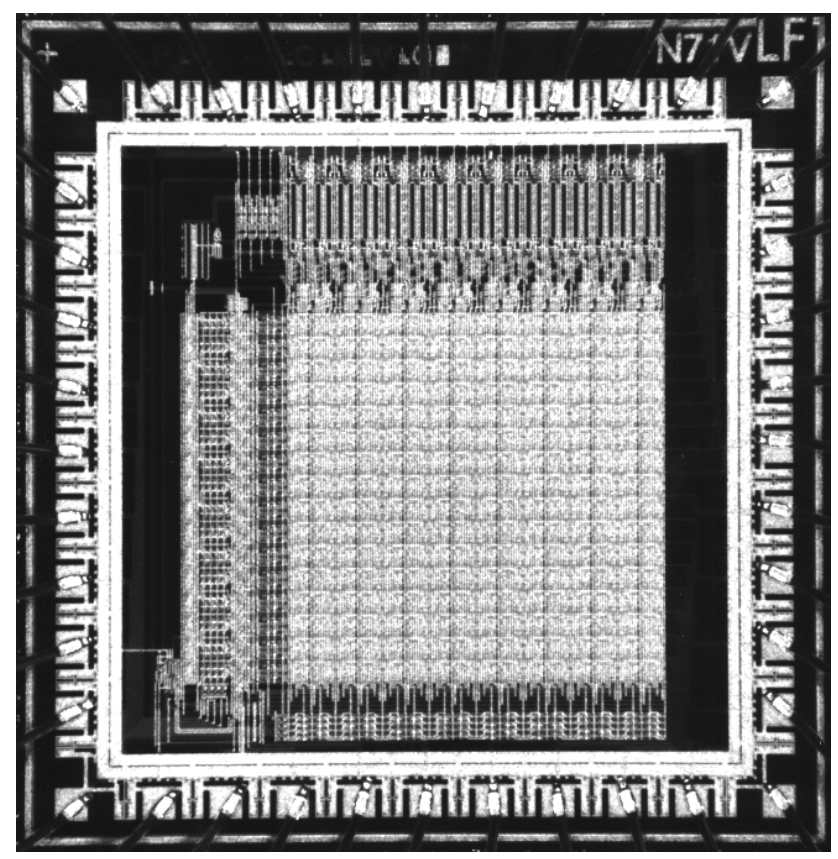

Figure 5: Chip micrograph of the $16 \times 16$ array, parallel learning vector quantizer. The die size is $2.2 \times 2.25 \mathrm{~mm}^{2}$ in $2 \mu \mathrm{m} C M O S$ technology.

\section{CONCLUSIONS}

We presented a parallel architecture and corresponding analog VLSI implementation of a learning vector quantizer, fabricated in a CMOS-compatible BiCMOS process. The chip is fully functional, and can be configured for neural variants on VQ such as Fuzzy ART and Kohonen self-organizing maps. The chip incorporates analog storage of the templates, sharing the same circuitry used for learning. With a dense cell size of $70 \times 80 \lambda$ units in scalable MOSIS technology, the integration of a 200-input, 1000-category classifier is feasible in a $0.35 \mu \mathrm{m}$ CMOS process.

\section{REFERENCES}

[1] A. Gersho and R.M. Gray, Vector Quantization and Signal Compression, Norwell, MA: Kluwer, 1992.

[2] Gail A. Carpenter, Stephen Grossberg and David B. Rosen, "Fuzzy ART: Fast Stable Learning and Categorization of Analog Patterns by an Adaptive Resonance System," Neural Networks, vol. 4, pp 759-771, 1991.

[3] B. Hochet, V. Peiris, S. Abdot, and M.J. Declercq, "Implementation of a Learning Kohonen Neuron Based on a New Multilevel Storage Technique," IEEE J. Solid-State Circuits, vol. 26, pp 262-267, 1991.

[4] W.C. Fang, B.J. Sheu, O.T.C. Chen and J. Choi, "A VLSI Neural Processor for Image Data-Compression Using SelfOrganization Networks," IEEE Transactions on Neural Networks, vol. 3 (3), pp 506-518, 1992.

[5] Y. He and U. Cilingiroglu, "A Charge-Based On-Chip Adaptation Kohonen Neural Network," IEEE Transactions on Neural Networks, vol. 4 (3), pp 462-469, 1993. 


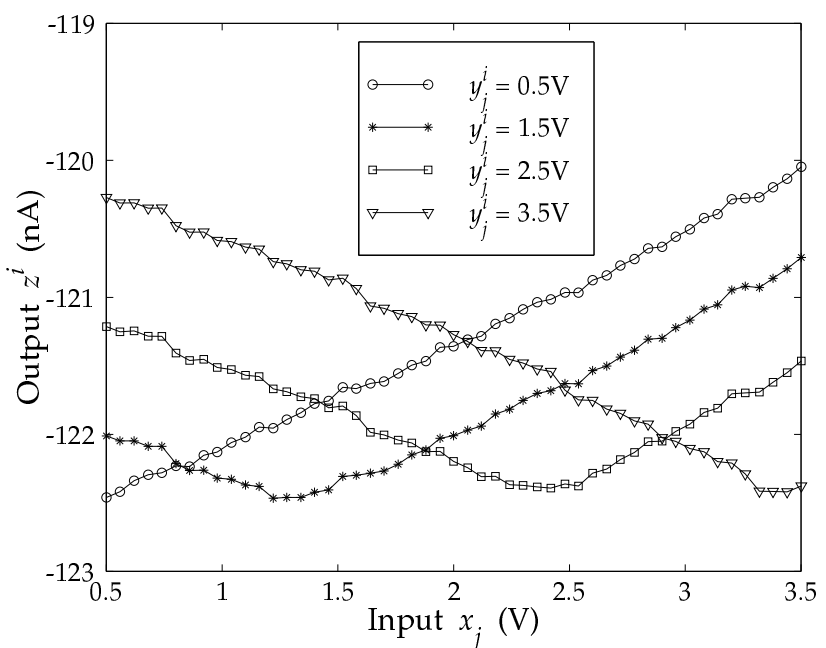

Figure 6: Measured distance output for one row of cells, sweeping one input component while fixing the other 15 inputs.

[6] G.T. Tuttle, S. Fallahi, and A.A. Abidi, "An 8b CMOS Vector A/D Converter," in ISSCC Technical Digest, IEEE Press, vol. 36, pp 38-39, 1993.

[7] G. Cauwenberghs and V. Pedroni, "A charge-based CMOS parallel analog vector quantizer," in Advances in Neural Information Processing Systems, Cambridge, MA: MIT Press, vol. 7, pp 779-786, 1995.

[8] M. Konda, T. Shibata, T. Ohmi, "Neuron-MOS Correlator Based on Manhattan Distance Computation for Event Recognition Hardware," in Dig. International Symposium on Circuits and Systems, Atlanta, GA, 1996.

[9] T. Serrano-Gotarredona, B. Linares-Barranco and J. L. Huertas, "A Real Time Clustering CMOS Neural Engine", $A d$ vances in Neural Information Processing Systems, vol. 7, Ed: D. S. Touretzky, Morgan Kauffmann, San Mateo, CA, 1995.

[10] J. Lazzaro, S. Ryckebusch, M.A. Mahowald, and C.A. Mead, "Winner-Take-All Networks of $\mathrm{O}(\mathrm{n})$ Complexity," in $\mathrm{Ad}$ vances in Neural Information Processing Systems, San Mateo, CA: Morgan Kaufman, vol. 1, pp 703-711, 1989.

[11] A.G. Andreou, K.A. Boahen, P.O. Pouliquen, A. Pavasovic, R.E. Jenkins, and K. Strohbehn, "Current-Mode Subthreshold MOS Circuits for Analog VLSI Neural Systems," IEEE Transactions on Neural Networks, vol. 2 (2), pp 205-213, 1991.

[12] G. Cauwenberghs and A. Yariv, "Fault-tolerant dynamic multi-level storage in analog VLSI," IEEE Transactions on Circuits and Systems II: Analog and Digital Signal Processing, vol. 41 (12), pp 827-829, 1994.

[13] G. Cauwenberghs, Analog VLSI Stochastic Perturbative Learning Architectures," Int. J. Analog Integrated Circuits and Signal Processing, vol. 13 (1/2), pp 195-209, 1997.

[14] G. Cauwenberghs, "A Micropower CMOS Algorithmic A/D/A Converter," IEEE Transactions on Circuits and Systems I: Fundamental Theory and Applications, vol. 42 (11), pp 913-919, 1995.

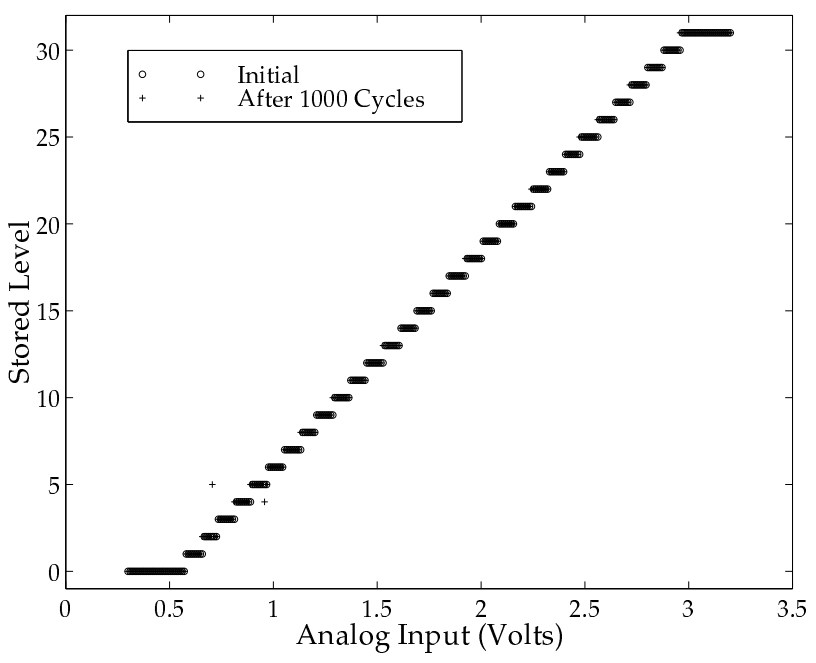

Figure 7: Stability of the analog memory array. Drift over 1000 self-refresh cycles, for 300 different initial conditions.

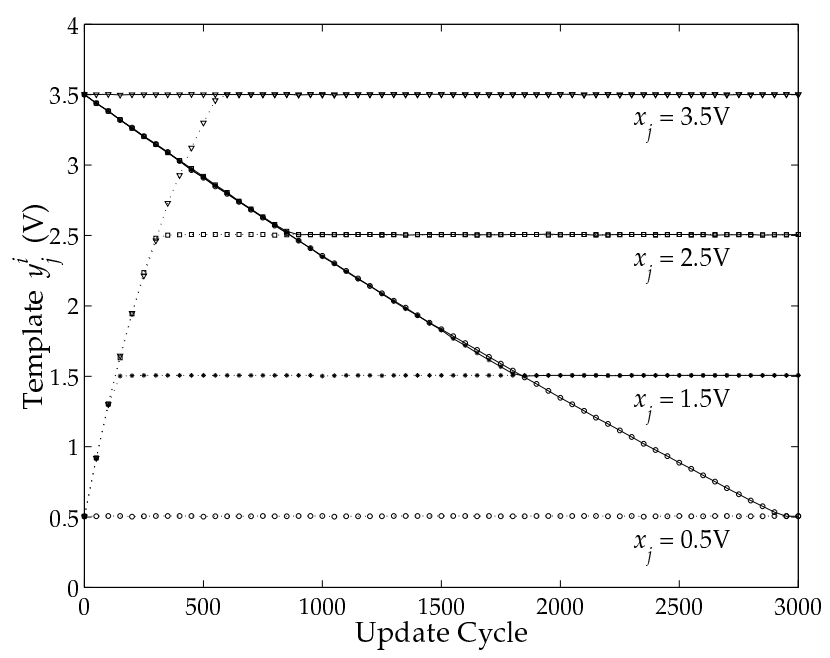

Figure 8: Template adaptation of VQ in learning mode, under fixed inputs, from low and high template initial conditions. 